\title{
Force Feedback in a Three-Dimensional Ultrasound-Guided Surgical Task
}

\author{
Christopher R. Wagner, Douglas P. Perrin, Robert D. Howe \\ Division Of Engineering And Applied Sciences \\ Harvard University \\ Cambridge, MA, USA \\ \{cwagner, dperrin, howe \\ @ deas.harvard.edu
}

\author{
Nikolay Vasilyev, Pedro J. del Nido \\ Department of Cardiac Surgery \\ Children's Hospital \\ Boston, MA, USA \\ \{Nikolay.Vasilyev, Pedro.DelNido\} \\ @ cardio.chboston.org
}

\begin{abstract}
Three-dimensional ultrasound ( $3 D$ US) is a novel imaging modality that allows real time visualization of internal body structures such as the heart, even through visually opaque blood and tissue. The real-time nature of $3 D U S$ allows minimally invasive manipulations to be carried out without an endoscopic camera. The quality of the images is not ideal, however, so other senses might be used to augment a surgeon's performance in a $3 D$ US-guided procedure. We investigate the combination of haptics under $3 D$ US in a force control task. Results suggest that stiffness of the environment plays a significant role as to the relative importance of vision versus haptics in this type of task.
\end{abstract}

\section{Introduction}

The use of 3D echo cardiography allows a number of minimally invasive surgical procedures to be carried out on a beating heart without the need of a endoscope. Because ultrasound imaging is not blocked by opaque blood, a number of procedures can thus be carried out without the use of bypass. One such procedure is the repair of an atrial septal defect (ASD), a hole in the atrial septum allowing blood flow between the right and left atrium. The current repair procedure we are developing involves anchoring a Dacron patch over the hole using expanding wire anchors [16]. The patch and anchors are placed using instruments that operate through small ports in the heart wall. This approach has been successfully demonstrated in in-vivo animal experiments. Successful deployment of the anchor is difficult; both proper positioning (localizing the anchor deployment tube over the patch and tissue surrounding the ASD) and proper force application (a force over a minimum threshold) are required for successful deployment (Fig. 1). We focus on the force application/regulation part of this task because the role of the sense of touch in surgery is still not well understood. Further complicating the situation is the imperfect visual information from the 3D US. Understanding how the tradeoff between visual information and haptic information relates to performance will allow better tools for surgeons, both in the form of visualization techniques and force feedback for surgical robotics.

Previous studies have investigated performance on tasks with combinations of visual and haptic feedback [14, 23, $18,12,6,9,1,20,5]$. An important difference here is the nature of the visual feedback. In most previous studies, the visual feedback takes more of the form of sensory substitution, with a graphical representation of a signal from a force sensor. Our study uses 3D US, whose rendering is a direct viewing of deformation (albeit degraded). An increased degree of processing is therefore necessary for subjects to extract force information. Furthermore, the amount of visual feedback of deformation and therefore force is different depending on the stiffness of the material deforming. Finally, the visual feedback in most of the previous studies returned absolute force information. Direct view only provides feedback on the relative force being applied.

We investigate the role of force feedback in the anchor deployment phase of ASD repair, a force control task under imperfect visual feedback (3D US). Force feedback is hypothesized to improve performance over only visual feedback based on the results of the above prior work. Understanding of the interaction between vision and the sense of touch is important because of the use of surgical robotics, where dexterity is enhanced but force feedback is lost [4]. Also, the understanding of how force feedback can improve performance when visual feedback is limited, as suggested by [5], would specify the necessary feedback quality for a given surgical task. We carry out a force control task mimicking the anchoring step in ASD repair to investigate these issues. 


\section{Methods}

This experiment investigates the ability of surgeons to regulate the interaction force between a surgical instrument and tissue using force feedback. The experiment mimics the surgical task of anchor deployment, where an anchor deployment tube is pressed against a patch covering tissue. The key factor to success in this type of task is proper regulation of the force between the tube and the patch/tissue. Forces that are too high cause the patch to laterally slide into the hole, resulting in the anchor grabbing the patch and not the tissue. Forces that are too low will result in failure of the anchor to puncture both patch and tissue, again resulting in an unsuccessful deployment. We attempt to encapsulate the force threshold part of the task.

Tasks are executed using 3D US as visual feedback to examine the effect of a novel and imperfect visualization modality on performance. Subjects carry out the same force regulation task with different force information feedback modalities to determine the ways in which force feedback may affect performance.

\subsection{Telemanipulation System}

The experiments use a laboratory teleoperation testbed consisting of two Phantom haptic interface devices (Model 1.5, SensAble Technologies, Inc., Woburn, Mass.) [2]. A teleoperation system is used to investigate different forms of force feedback while maintaining the same interface. One Phantom acts as the surgeon master controller and the other acts as the surgical robot. The master is an unmodified Phantom with the stylus attachment. Subjects control the motion of the surgical robot by moving the stylus, held in a pen grasp, where the tip of the stylus maps to the proximal end of the instrument shaft. The port was placed at the middle of the length of the instrument shaft so that motions of the hand were the same scale as instrument motions. The surgical instrument used was a tube (14 gauge blunt needle,

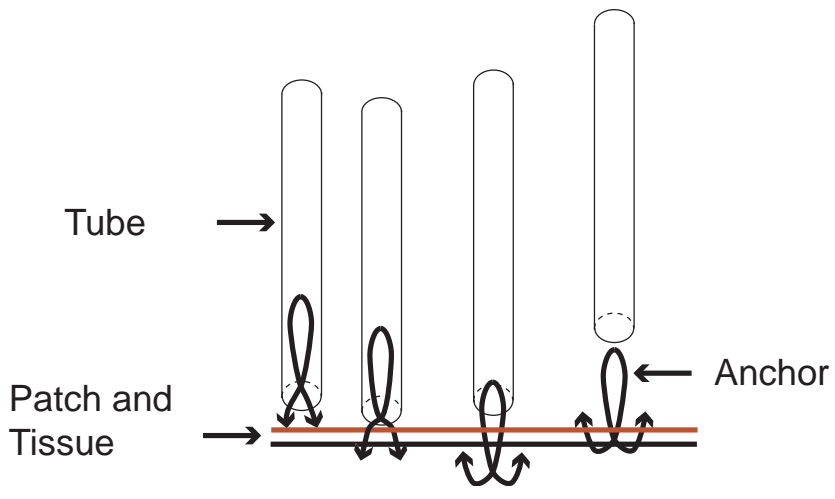

Figure 1. Anchor deployment in ASD repair

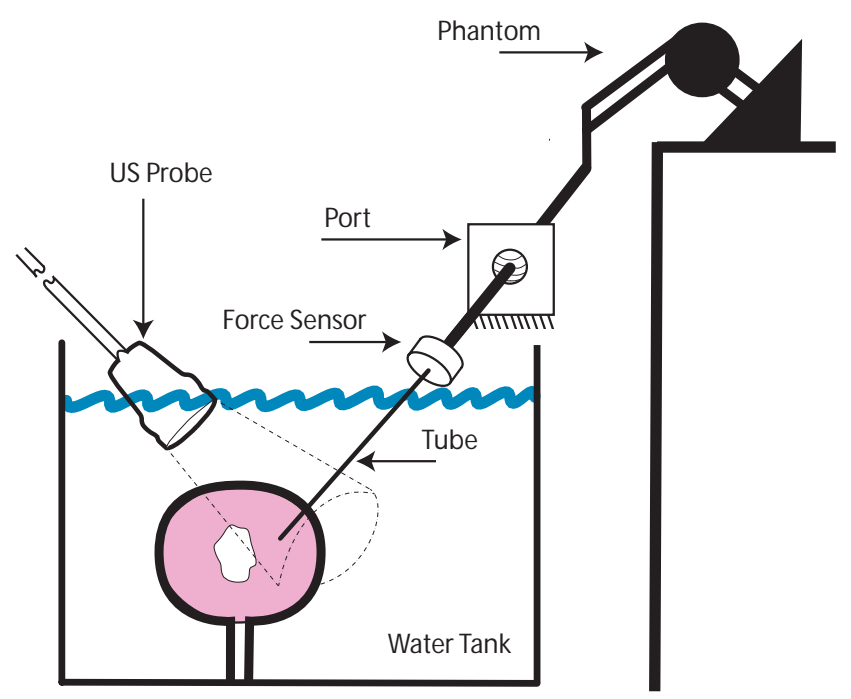

Figure 2. Surgical robot and surgical environment

diameter $2.1 \mathrm{~mm}$ ) identical to the one on the anchor deployment device (Fig. 2).

Axial forces (along the shaft of the instrument) are sensed by a one-axis force sensor with an RMS noise level of $0.01 \mathrm{~N}$ (LCFD-1KG, Omega Engineering, Inc., Stamford, CT) built into the instrument shaft. The surgical robot is controlled with a standard position feedforward scheme traditionally used in teleoperated systems [13]. When in force feedback mode, the Phantom control computer samples the axial forces at $1 \mathrm{kHz}$ and transforms the forces to the proximal end of the shaft, removing the transmission of friction forces at the port.

The teleoperation system, including the master, the surgical robot, and the force sensor, are controlled by a 2.0 $\mathrm{GHz}$ Athlon computer running Windows XP. The surgical robots position is controlled using proportional position/velocity control, independent of force feedback, with gains $k_{p}=0.2 \mathrm{~N} / \mathrm{mm}$ and $k_{d}=0.00035 \mathrm{Ns} / \mathrm{mm}$. These values were empirically derived to provide uniform stiffness in the portion of the workspace used for these trials while maintaining stability of the teleoperation system [2]. The control algorithm is implemented in Visual C++ along with the force sensor interface. All forces and positions were logged at $1 \mathrm{kHz}$.

\subsection{Visual Feedback}

The subjects received visual feedback from a 3D US system (Sonos 7500, Philips Medical Systems, Andover, MA) (Fig. 3). The system records three dimensional volume information using a phased array ultrasound transducer then renders a $2 \mathrm{D}$ view for display. Objects of different me- 
chanical impedance are rendered with different intensities. Thresholding allows segmentation of these objects from the rendered view, thus opaque blood can appear transparent while tissue is still visible. Image quality is not perfect, however, as the segmentation introduces irregularities at surface boundaries. Also, rigid instruments introduce scattering and shadowing artifacts to the visual display because of the high mechanical impedance. The anchor deployment tube was coated with a low impedance plastic to reduce these effects.

To minimize the mental effort of relating visual and instrument frames, the rendering view was chosen to match closely with the relative positioning of the master and display [19]. The view used remained constant across all trials and subjects. The rendered view displayed a volume of 3 $\mathrm{cm} \times 3 \mathrm{~cm} \times 2 \mathrm{~cm}$ with a voxel resolution of approximately $0.5 \mathrm{~mm}$.

The experiment was carried out in a water tank to allow use of the US. The probe was mounted at a 30-degree angle to the tissue to allow full visualization while not interfering with the motion of the instrument.

\subsection{Force and Vibration Feedback}

Three modalities of force information feedback were used during this experiment. The first was only the rendered view from the ultrasound (US); subjects had to determine the level of force application based on the observed deformation. The second modality was the US view combined with force feedback (US+FF). The surgical master would push back on the subject's hand with a force proportional to the one sensed by the force sensor. That force is scaled by a $75 \%$ gain, chosen to provide an intuitive level of force to surgeons for this specific experiment.

The third modality was US view combined with a tactile vibration (US+V) that was chosen to give near-optimal feedback on the correctness of the force being applied without applying a net force to the hand or causing a gaze shift in the subject. The vibration force commanded to the master was

$F_{v}(t)=\left\{\begin{array}{cc}0, & F_{a}(t)<1.5 \\ 0.2+0.4\left(F_{a}(t)-1.5\right) \sin (500 \pi t) & F_{a}(t) \geq 1.5\end{array}\right.$

where $F_{a}(t)$ is the measured contact force. This caused the user to feel a vibration as soon as the proper threshold was reached and give a scaling cue if the user continued to apply a force over the threshold. The vibration in the motors also manifested as an easily perceptible auditory cue. From pilot studies, users were easily able to apply $1.5 \mathrm{~N}$ of force with high accuracy and precision with this feedback modality.

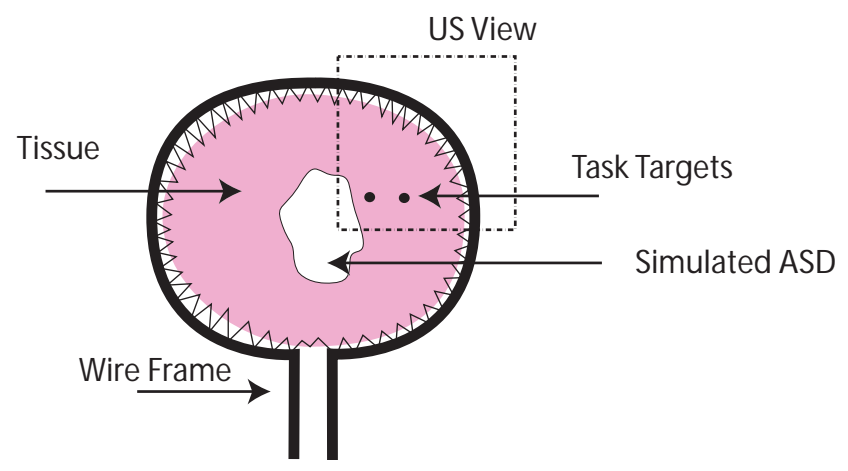

\section{Figure 4. Tissue with target positions for force application}

\subsection{Tissue target}

An excised porcine atrial septum was used to match the tissue mechanics during an actual surgery while while maintaining repeatability. The heart tissue was mounted to a rigid wire frame $4 \mathrm{~cm}$ in diameter for ease of positioning for the experiment (Fig. 4). While not directly used in the study, an artificial defect ( $8 \mathrm{~mm}$ diameter) was created by excising the central part of the septum to mimic the true environment and provide a recognizable landmark in the US view. The tissue was harvested 2 months prior to the experiment, drained of blood, and kept viable in a $10 \%$ Formalin solution. The same tissue was used for all trials.

Two positions on the tissue were chosen as targets for the force application. More than one target position was used so subjects did not anticipate and remember the exact hand motion necessary to execute the task. Also, as stiffness of the tissue may impact both the force and visual feedback [5], positions of different stiffnesses were chosen by choosing positions with different distances from the ASD (Fig. 4). The measured stiffnesses of the lower stiffness target position (closer to the ASD) and the higher stiffness target position (farther from the ASD) were $160 \mathrm{~N} / \mathrm{m}$ and 240 $\mathrm{N} / \mathrm{m}$, respectively.

The patch and the anchor deployment system are not included in this experiment to reduce the number of variables. Only the deployment tube and tissue are used to investigate the key factor of accurate force application. Without the patch, the optimal force needed to ensure proper anchor deployment while avoiding puncture (as determined by pilot studies) is $1.5 \mathrm{~N}$.

\subsection{Protocol}

Subjects were instructed to move the deployment tube to the correct position, then apply the tube against the tissue with the correct amount of force $(1.5 \mathrm{~N})$. Subjects then 

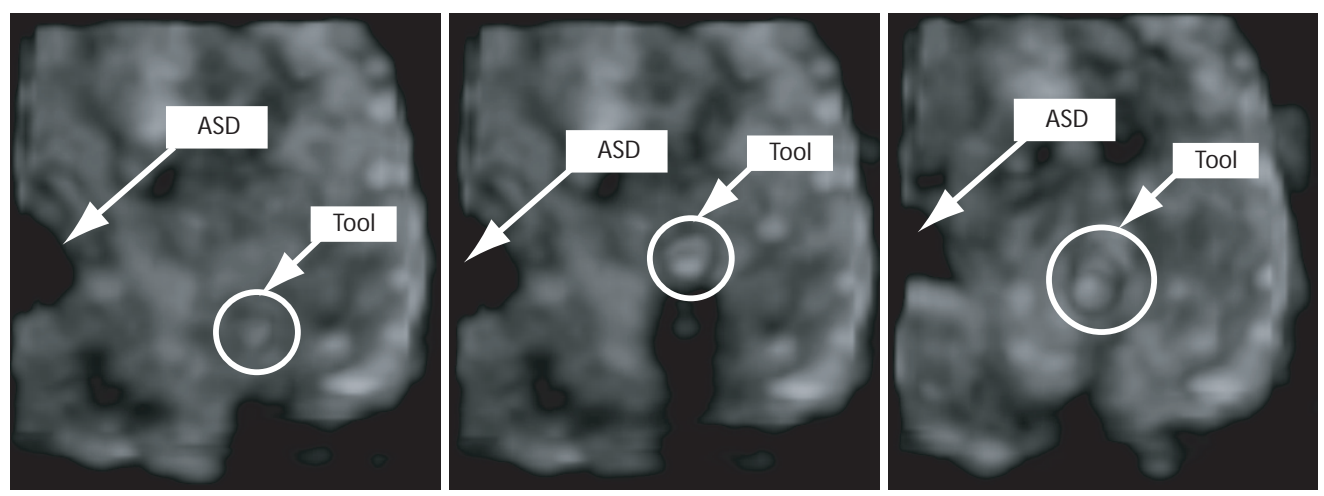

Figure 3. US images for the start, middle, and end of a trial

held down a button on the stylus interface for one second to signal when they felt the correct force amount was being applied. These trials were executed under three different forms of force information and at two different locations on the tissue. Before each trial, subjects were informed of the force information condition (US, US+FF, or US+V) and the desired position (closer to or farther from the ASD). The accuracy of the force application was the only error criterion given to subjects (subjects were not asked to trade off speed for accuracy).

Subjects trained for approximately 10 minutes to familiarize themselves with the teleoperation system and to learn the feedback when applying the correct force under different feedback conditions. During training for the US and the US+FF cases, subjects were verbally informed when the $1.5 \mathrm{~N}$ threshold was reached. For the US+V case, subjects learned to apply the lowest amount of force such that the vibration occurred. Under all cases (feedback and position), subjects trained until the threshold could reliably be applied three times in a row.

Eight surgeons were subjects, all with backgrounds in minimally invasive surgery (more than 3 years) but having little experience with manipulation under 3D US. The range of backgrounds varied from surgical residents to attending surgeons. Each subject performed 5 trials of each combination of feedback type and target position, for a total of 30 trials per subject.

\subsection{Measures}

Four different outcome measures were examined for each trial to characterize the performance of a subject: the mean force during the final second; the coefficient of variation of each subject's final second mean force (standard deviation divided by the mean); the rate of a successful anchor placement (whether the final second mean force was within $+/-0.5 \mathrm{~N}$ of the target force); and the trial time. The coefficient of variation was included to examine sub- jects' repeatability in force application (precision) without the scaling effect of mean. Even though time was not told to the subjects as a specific error measure, time is included to examine whether subjects were trading off time with force.

\subsection{Statistical Analysis}

To determine statistical significance of our experimental conditions we used a repeated measures ANOVA with within subject variables of force feedback condition and stiffness condition. The statistical analysis included success rate, mean force, coefficient of variation, and time. The SPSS statistical analysis software package (Version 13.0, SPSS Inc., Chicago, Ill.) was used to carry out the analysis. A $p$ value of less than 0.05 was considered statistically significant.

\section{Results}

Success on a trial was evaluated by determining if the mean force in the last second of each trial (during which time the subject was pressing the stylus button) was within $+/-0.5 \mathrm{~N}$ of the target application force of $1.5 \mathrm{~N}$ (Fig. $5)$. Feedback condition significantly influenced success rate $(F(2,14)=9.07, p<0.02)$, with the US+V feedback resulting in the highest average success rate of $96 \%$. The main effect of stiffness did not significantly affect success rate $(F(1,7)=0.004, p>0.9)$ because the effect was opposite for the US and US+FF cases. Thus, the significant effect of stiffness manifested in the interaction term between stiffness and feedback type $(F(2,14)=8.95, p<0.02)$. Average success rates of approximately $56 \%$ were achieved under the low stiffness, US and the high stiffness, US+FF cases. For the other cases of high stiffness, US and low stiffness, US+FF the average success rate was below $38 \%$.

Mean force applied during the last second of each trial (Fig. 6) was significantly lower for low stiffness targets 


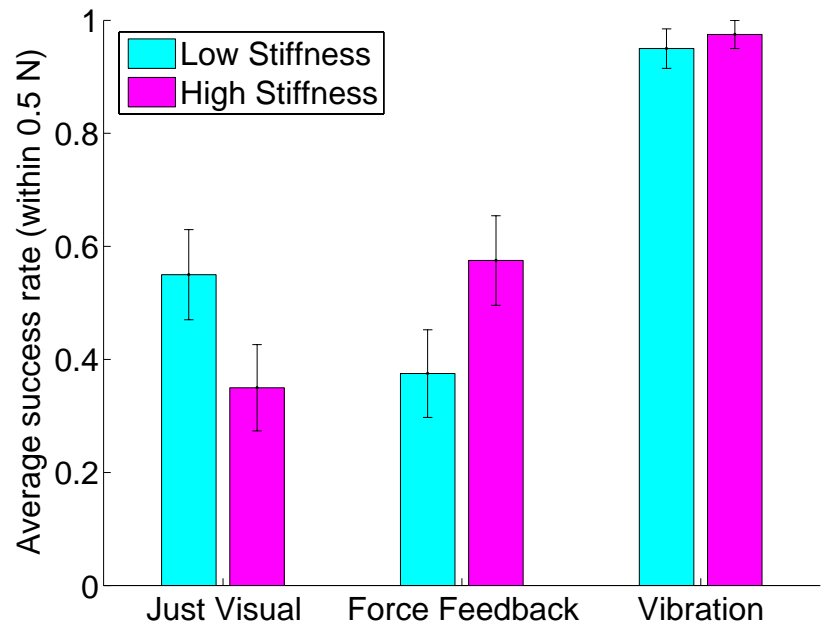

Figure 5. Success rate (within $0.5 \mathrm{~N}$ of target). Error bars show standard error.

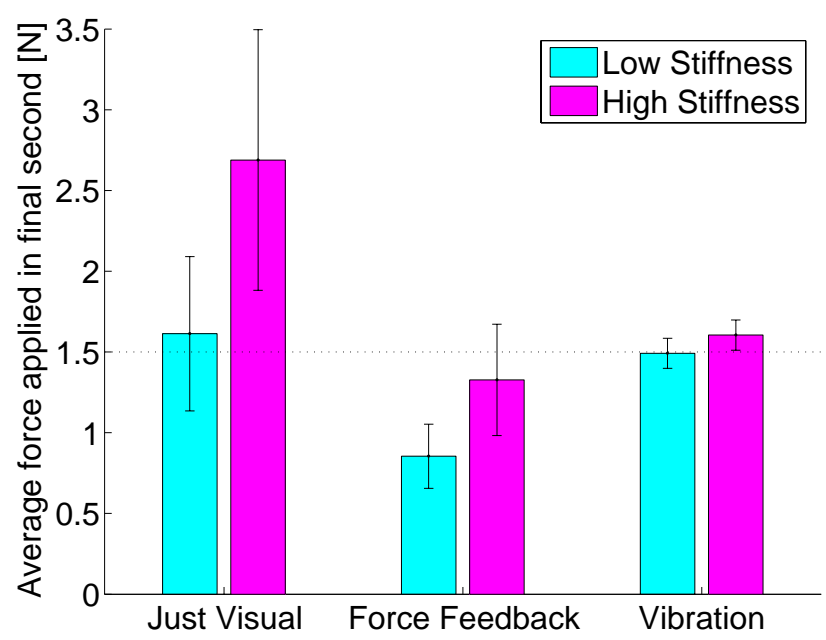

Figure 6. Mean force for different conditions. Dotted line shows target force and error bars show $95 \%$ confidence interval of mean estimation.

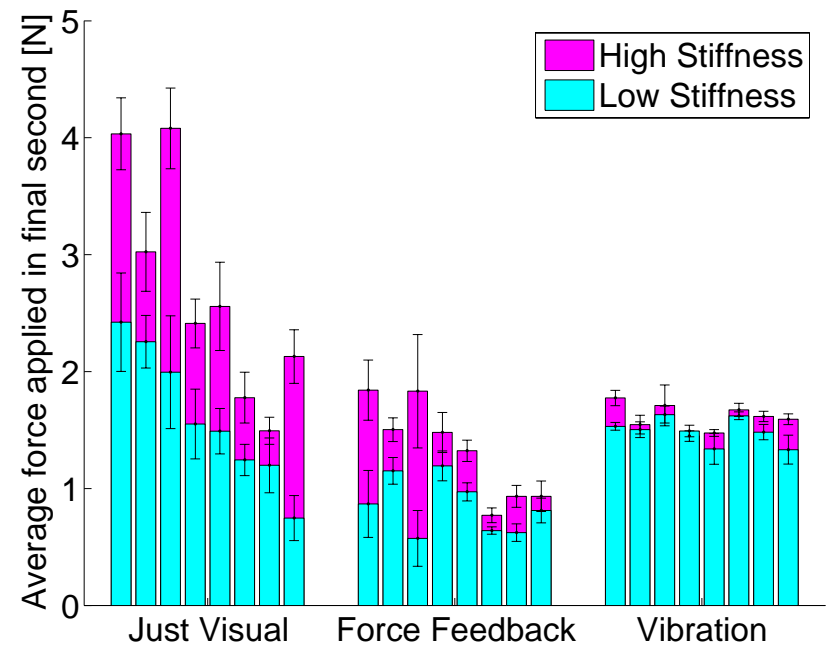

Figure 7. Mean force for each subject. Subjects are in same order for all three conditions. Error bars show standard error.

$(F(1,7)=22.8, p<0.002)$. Feedback condition also significantly affected mean force $(F(2,14)=15.68, p<0.005)$. The highest average forces were applied under the US feedback, where mean forces for both the low and high stiffness cases were above the target force of $1.5 \mathrm{~N}$. Forces were consistently below the target force when subjects received US+FF feedback, applying an average of $0.85 \mathrm{~N}$ and $1.32 \mathrm{~N}$ for the low and high stiffness cases, respectively. Subjects were most accurate with the US+V feedback case, with both means being within $0.1 \mathrm{~N}$ of the target force. Intersubject variation is highest with only ultrasound feedback (Fig. 7) and minimal with the vibration feedback.

Coefficient of variation of the mean force applied during the last second was analyzed to determine the effect of feedback and stiffness on precision of force application (Fig. 8). A lower stiffness target position significantly increased the coefficient of variation $(F(1,7)=9.25, p<0.02)$. Feedback condition also significantly affected the coefficient of variation $(F(2,14)=9.12, p<0.02)$, with the vibration feedback case having coefficients at least twice as small as the other two conditions. A pairwise comparison, however, reveals that the only two significantly different feedback conditions were US and US+V $(p<0.001))$. The difference between $\mathrm{US}+\mathrm{FF}$ and $\mathrm{US}+\mathrm{V}$ almost reached significance $(p=0.051)$.

Average time needed to complete a trial was analyzed to assess any performance tradeoffs (Fig. 9). Having a low stiffness target position significantly reduced the average time spent per trial $(F(1,7)=12.9, p<0.01)$, reducing time by up to 5.3 seconds in the US+V feedback condition. Feedback condition did not significantly affect trial 


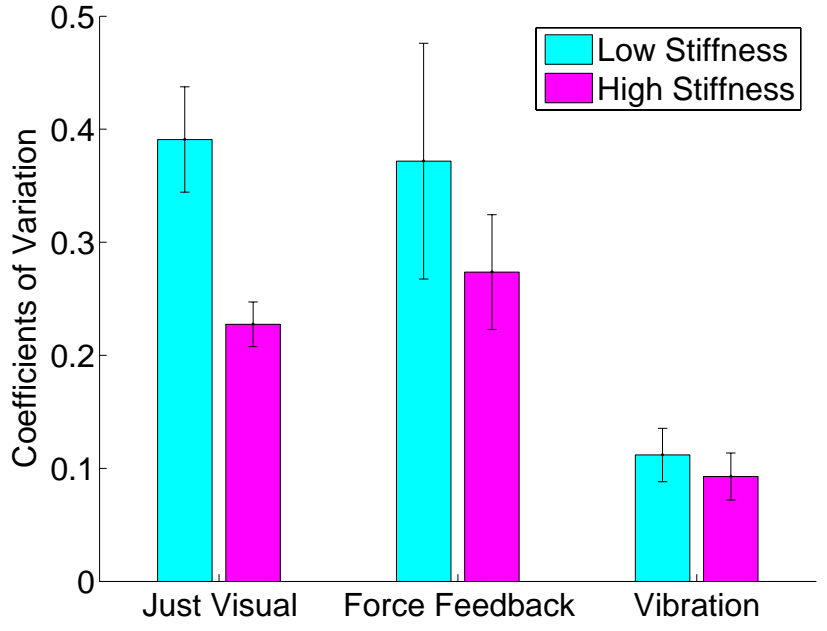

Figure 8. Average coefficient of variation. Error bars show standard error.

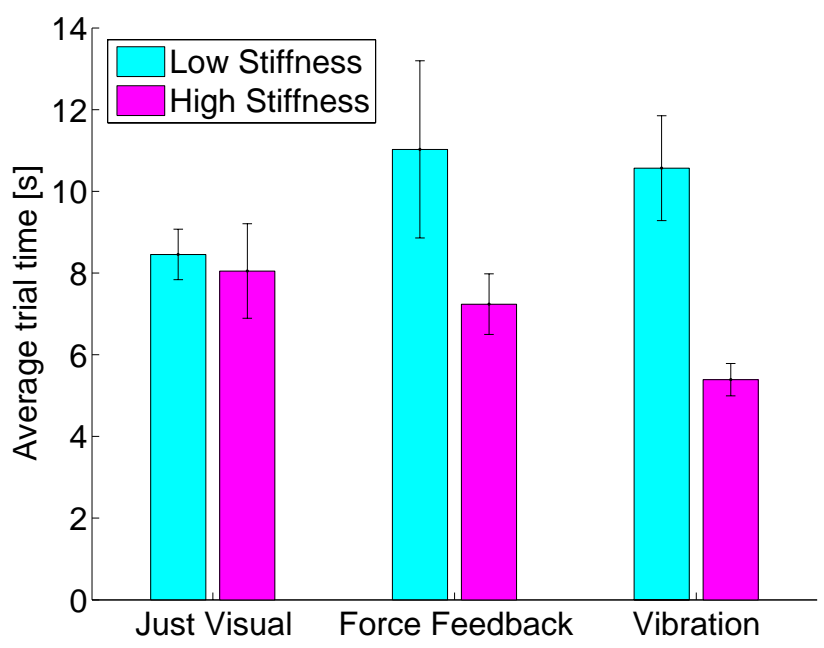

Figure 9. Average trial time. Error bars show standard error. time $(F(2,14)=0.94, p=0.365)$, with all feedback conditions averaging from 7 to 10 seconds per trial.

\section{Discussion}

In this experiment we examined the hypothesis that the addition of force feedback to a force application task when visual information is suboptimal would result in improved performance. Our results suggest that performance is dependent on the stiffness of the object being pushed and that the addition of force feedback does not improve performance nearly as much as an indication of the specific target force level.

A goal of this study is to understand the tradeoff between vision and haptics as they relate to performance in a force control task. A number of previous studies have established the degree of force control precision (using visual feedback of force) of the finger [14] [23], elbow and wrist [18], or with a probe [12]. Jones demonstrated that the feedback of a visual representation of force improves accuracy and precision on a force control task over force feedback alone [6]. These studies, however, used a visual representation of force more akin to sensory substitution. While sensory substitution has been shown to aid performance in a force control knot tying task [9], little work has been done examining the tradeoffs between force feedback and direct vision in a force control task (the situation most often encountered in surgery). Cao showed that direct view of deformations can provide force information and associated performance benefits on a simulated task [1]. Desai investigates direct view of deformations in conjunction with force feedback, but only for the identification of stiffnesses [20]. Finally, Gerovich et al examine visual and haptic feedback in a position control task (where the visual feedback is simulated deformation) and find that force feedback may not be necessary unless visual feedback is limited [5].

A recent hypothesis as to the relationship of vision and the sense of touch is that the central nervous system uses maximum likelihood estimation (MLE) to determine dominance [3]. Therefore, the sense with the lowest variance in the estimate of the salient parameter is weighted more than the other sense. Because there is a clear dominance issue in our results (in some cases, subjects do worse when force feedback information is added), we will discuss our results in the context of maximum likelihood estimation and information.

This framework leads to a hypothesis of why subjects performed better with just US in the low stiffness case than the high stiffness case. With low stiffness, for a small change in force, there is a large visual deformation. Conversely, with high stiffness, a small force of the deployment tube against the tissue will only result in a small visual deformation. Thus, when interacting with objects of differing 
stiffnesses, objects with lower stiffness will have more information in the visual channel. A reason that the forces were higher in the high stiffness case is that subjects had to apply a certain deformation to get a visual signal out of the noise; that same deformation will result in higher forces for a high stiffness tissue.

The MLE theory can also account for why the US case performed well in the low stiffness case and the force feedback helped in the high stiffness case. This could be explained by dominance-when force feedback is added, force dominates because of the perceived low variance in the force signal. In the high stiffness case, there is a large amount of signal in the force feedback channel while there is relatively little in the visual, so the dominance is correct which results in an increase in performance. An alternative explanation is that mechanical work is the salient parameter governing this force control task, taking into account the displacement over which a force is applied, as investigated by [17].

An explanation of the low overall forces in the force feedback condition can be given by extending this statistical framework to include expectation and prior knowledge. Previous work has shown that the central nervous system behaves according to a Bayesian framework during force control tasks, integrating a prior expectation of force with current sensation for control [10]. Work in softness discrimination has also shown this anticipatory behavior [11]. The low forces observed with the US+FF condition may be a result of surgeons' prior expectations of the forces encountered in surgical tasks. Another explanation, also proposed by [10], is that subjects trade off force control correctness with force control effort. At high forces, the effort needed becomes significant enough to affect subjects' idea of the target force.

\subsection{Application to surgery}

Several differences exist between this study and an actual 3D US-guided ASD repair. A primary difference is that we are using excised, static tissue when the real surgery is carried out on living, dynamic tissue. The use of the formalin solution to preserve the tissue also stiffens the tissue somewhat, changing the mechanical interaction. Surgeons may also be using different expectations and training for force interaction with tissue due to the interface of the surgical robot because different muscle groups are utilized [7].

Another difference between this study and actual surgery is the quality of the visual feedback. During surgery, the relative position of the heart and the ultrasound probe can change with the beating of the heart. Often times, the ASD moves out of the scope of the probe and needs to be relocated. This effect, combined with the complexity of patch deployment, temporary occlusions by tools, and noise intro- duced by fluid flow all serve to degrade the information in the visual channel [15]. Returning to the statistical framework, because the variance in the visual channel information is higher, other forms of informational feedback may be more helpful and would dominate.

The benefit of force or any type of informational feedback is clearly related to the task and the consequenses of error conditions. For instance, in the anchor deployment task, overapplication of force could result in a puncture. Therefore, force feedback (which minimizes peak forces [22]) can improve overall performance. Another example of correct feedback is in applying optimal suture tying forces using a robot; when the subject receives direct feedback of suture forces through sensory substitution the force accuracy is higher than even through direct contact [9].

That being said, a final point to address is the feasibility/ease of use of any feedback used to augment surgical performance. For instance, even though the vibration feedback allowed subjects to accurately and precisely control tool tip force, most surgeons preferred the force feedback. One reason given is that the vibration was distracting; it was difficult to pay attention to anything else. In surgery, when a number of complex motions need to coordinate to a range of subtle cues, the presence of one powerful cue may wash out other subtler cues. Potentially, there is no cognitive workload imposed by a natural feedback such as force feedback. Another hypothesis is that surgeons are used to having forces and use of the robot with just visual feedback removed all force feedback. In either case, force feedback has been shown to be useful across a number of surgical tasks $[22,8,20]$, while the vibration feedback given here is useful in just this one task.

The results from this experiment lead to a number of interesting questions as to the benefit of different forms of feedback in surgery. An obvious hypothesis just mentioned is mental workload of different forms of feedback in surgery. Force feedback is costly and not necessarily a perfect information source for all tasks, but it can potentially provide benefit (both passive and informational [21]) at little cost to cognitive demand because of its intuitive nature.

\section{References}

[1] C. Cao, J. Webster, J. Perreault, S. Schwaitzberg, and G. Rogers. Visually perceived force feedback in simulated robotic surgery. In Proceedings of the 47th Annual Meeting of the Human Factors and Ergonomics Society, pages 1466-1470, 2003.

[2] M. C. Cavusoglu, D. Feygin, and F. Tendick. Critical study of the mechanical and electrical properties of the phantom haptic interface and improvements for high performance control. Presence: Teleoperators and Virtual Environments, 11:555-568, 2002. 
[3] M. Ernst and M. Banks. Humans integrate visual and haptic information in a statistically optimal fashion. Nature, 6870(415):429-433, Jan 2002.

[4] V. Falk, J. McLoughin, G. Guthart, J. S. Jr, T. Walther, J. Gummert, and F. Mohr. Dexterity enhancement in endoscopic surgery by a computer controlled mechanical wrist. Minimally Invasive Thoracic and Allied Technology, 8(4):235-242, 1999.

[5] O. Gerovich, P. Marayong, and A. Okamura. The effect of visual and haptic feedback on computer-assisted needle insertion. Computer Aided Surgery, 6(9):243-249, 2004.

[6] L. Jones. Visual and haptic feedback in the control of force. Experimental Brain Research, 2(130):269-272, Jan 2000.

[7] L. Jones. Perceptual constancy and the perceived magnitude of muscle forces. Experimental Brain Research, 2(151):197-203, Jul 2003.

[8] A. Kazi. Operator performance in surgical telemanipulation. Presence, 10:495-510, 2001.

[9] M. Kitagawa, D. Dokko, A. Okamura, and D. Yuh. Effect of sensory substitution on suture-manipulation forces for robotic surgical systems. Journal of Thoracic Cardiovascular Surgery, 1(129):151-8, Jan 2005.

[10] K. Kording, S. Ku, and D. Wolpert. Bayesian integration in force estimation. Journal of Neurophysiology, 5(92):31613165, Nov 2004.

[11] R. LaMotte. Softness discrimination with a tool. Journal of Neurophysiology, 83:1777-1786, 2000.

[12] S. Lederman, R. Howe, R. Klatzky, and C. Hamilton. Force variability during surface contact with bare finger or rigid probe. 12th International Symposium on Haptic Interfaces for Virtual Environment and Teleoperator Systems, pages 154-160, 2004.

[13] T. B. Sheridan. Telerobotics, Automation, and Human Supervisory Control. Cambridge, Mass.: MIT Press, 1992.

[14] J. shing Chen. Human Haptic Interaction with Soft Objects: Discriminability, Force Control, and Contact Visualization. PhD dissertation, Massachusetts Institute of Technology, Department of mechanical engineering, 1996.

[15] J. Sosnoff and K. Newell. Intermittent visual information and the multiple time scales of visual motor control of continuous isometric force production. Perception and psychophysics, 2(67):335-344, Feb 2005.

[16] Y. Suematsu, G. Marx, J. Stoll, P. DuPont, R. Cleveland, R. Howe, J. Triedman, T. Mihaljevic, B. Mora, B. Savord, I. Salgo, and P. del Nido. Three-dimensional echocardiography-guided beating-heart surgery without cardiopulmonary bypass: a feasibility study. Journal of Thoracic Cardiovascular Surgery, 4(128):579-587, Oct 2004.

[17] H. Tan, N. Durlach, G. Beauregard, and M. Srinivasan. Manual discrimination of compliance using active pinch grasp: the roles of force and work cues. IPerception and Psychophysics, 4(57):495-510, 1995.

[18] H. Z. Tan, M. A. Srinivasan, B. Eberman, and B. Cheng. Human factors for the design of force-reflecting haptic interfaces. ASME Dynamic Systems and Control, 55(1):353-359, 1994.

[19] F. Tendick, R. Jennings, G. Tharp, and L. Stark. Sensing and manipulation problems in endoscopic surgery. Presence, 2:66-81, 1993.
[20] G. Tholey, J. Desai, and A. Castellanos. Force feedback plays a significant role in minimally invasive surgery: results and analysis. Annals of Surgery, 1(241):102-109, Jan 2005.

[21] C. Wagner and R. Howe. Mechanisms of performance enhancement with force feedback. First Joint EuroHaptics Conference and Symposium on Haptic Interfaces for Virtual Environment and Teleoperator Systems, pages 21-29, 2005.

[22] C. Wagner, N. Stylopoulos, and R. Howe. The role of force feedback in surgery: Analysis of blunt dissection. Haptics Symposium, pages 73-79, 2002.

[23] M. Wu, J. J. Abbott, and A. M. Okamura. Effects of velocity on human force control. First Joint Eurohaptics Conference and Symposium on Haptic Interfaces for Virtual Environment and Teleoperator Systems (World Haptics), pages 73-79, 2005. 